\title{
The Unexpected Advantages of Using D-Amino Acids for Peptide Self- Assembly into Nanostructured Hydrogels for Medicine
}

\author{
Michele Melchionna ${ }^{\mathrm{a}}$, Katie E. Styan ${ }^{\mathrm{b}}$, and Silvia Marchesan ${ }^{\mathrm{a},}$
}

${ }^{a}$ Department of Chemical and Pharmaceutical Sciences, University of Trieste, Via Giorgieri 1, 34127 Trieste, Italy; ${ }^{b}$ CSIRO Manufacturing Flagship, Clayton, VIC 3168, Australia

\begin{abstract}
Self-assembled peptide hydrogels have brought innovation to the medicinal field, not only as responsive biomaterials but also as nanostructured therapeutic agents or as smart drug delivery systems. D-amino acids are typically introduced to increase the peptide enzymatic stability. However, there are several reports of unexpected effects on peptide conformation, self-assembly behavior, cytotoxicity and even therapeutic activity. This mini-review discusses all the surprising twists of heterochiral self-assembled peptide hydrogels, and delineates emerging key findings to exploit all the benefits of D-amino acids in this novel medicinal area.
\end{abstract}

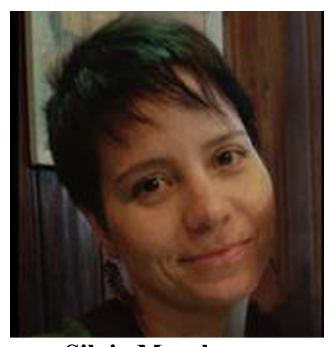

Silvia Marchesan

Keywords: Chirality, D-Amino acids, Enantiomers, Heterochiral, Hydrogels, Nanomaterials, Peptides, Self-assembly.

\section{INTRODUCTION}

Nanomaterials are revolutionizing medicine in the $21^{\text {st }}$ century by providing novel properties and introducing new therapeutic and diagnostic paradigms [1]. The rise of selfassembling peptide nanostructures [2] and hydrogels has brought innovation to various medicinal fields [3], spanning from adaptive [4], biodegradable tissue engineering scaffolds [5] for wound healing and cell encapsulation, to unprecedented paradigms in drug delivery, and even targeted cell therapy [6]. Among the many peptide-based systems, lowmolecular-weight gelators deserve special mention as they possess an economic advantage over longer peptides or more complex peptide-based derivatives [7].

There are many peptide-based systems that exploit various interactions, such as electrostatic interactions, aromatic $\pi-\pi$ stacking, hydrogen bonding, etc., to drive self-assembly and these have been extensively reviewed elsewhere [3]. These nanomaterials have well-established advantages as is apparent from the majority having been patent-protected and some constituting the core technology of spin-off companies [8].

The incorporation of unnatural D-amino acids in the selfassembling peptide motifs is an aspect that is attracting more and more attention owing to the many, often unexpected, effects. From a medicinal perspective, D-amino acids have very interesting features. For example, it is well-known that incorporation of D-glutamic acid and D-alanine into the bacterial cell wall protects it against degradation by exogenous proteases [9]. Likewise, albeit less well known, D-serine and D-aspartic acid regulate neurotransmission in the brain,

\footnotetext{
*Address correspondence to this author at the Department of Chemical and Pharmaceutical Sciences, University of Trieste, 34127 Trieste, Italy; Tel. +39-040-558-3923; Fax. +39-040-558-3903. E-mails: smarchesan@units.it, marchesan.silvia@gmail.com
}

where they occur naturally [10]. Insertion of D-amino acids into heterochiral peptides is also a convenient approach to generate bioactive motifs to promote cell adhesion [11], and to control stem cell differentiation [12]. Chirality effects at the supramolecular level are far from well understood, and those related to interactions with biological entities, or therapeutic effects, can be surprising and unanticipated. This aspect of biomaterials research is only just emerging but is evolving very rapidly.

In this mini-review we discuss key findings thus far and comment on how we view the future directions of such an innovative area of research. We discuss only those peptides that contain D-amino acids and form macroscopic hydrogels, since we are interested in the intentional design and use of sequences that self-assemble into hierarchically nanostructured, macroscopic materials. Sequences that display only limited self-assembling ability, leading to isolated nanostructures, have been excluded.

In more detail, the focus of this mini-review is the various effects and advantages of introducing D-amino acids on self-assembling peptides for biomedical use. First, we discuss enzymatic stability towards, for example, proteases, isomerases, racemases, and phosphodiesterases, Second, we analyze the effects on peptide conformation, and especially on secondary structures that are modified by adding, or even intentionally designed to include, D-amino acids at specific positions. Third, we highlight the supramolecular behavior leading to peptide self-assembly and gelation. Finally, we summarize the often unanticipated therapeutic effects that follow upon introduction of D-amino acids.

\section{THE ADVANTAGE OF ENZYMATIC STABILITY}

From a medicinal chemist's perspective, the most obvious reason to use D-amino acids for biomaterials is their theoretically lessened susceptibility to enzymatic processing, 
and thus their prolonged stability and function in a biological environment. We say "theoretical" because although it is generally accepted that D-peptides resist the action of proteases relative to their L-analogues, there are surprising examples of other enzymes that can act upon D-amino acids at the same rate as L-amino acids [13].

Nevertheless, early reports on the use of D-amino acids in self-assembled hydrogel biomaterials were conducted under the hypothesis of higher stability against enzymatic degradation. While it may be sufficient to incorporate just a few Damino acids $[6,14]$, the typical approach consists of using the full D-enantiomer of an established self-assembling motif, i.e. substituting all L-amino acids with their Dcounterparts [15]. Increased proteolytic stability is often just assumed [16], and only rarely tested, and even with limited enzymes such as the most often selected proteinase K [17]. Alternatively, chymotrypsin, collagenase, cathepsin B, or matrix metalloproteinases (MMP) are useful enzyme models [18]. Overall, increased enzymatic stability towards the action of peptidases or proteases has been shown in several cases, with differences in degradation rates expectedly varying dependent on sequence and position of the D-amino acid(s), as well as conditions applied [19]. From a medicinal perspective, inert bulking agents have value, however a greater advantage comes when the increased stability is coupled to a therapeutic or diagnostic use, such as the delivery of drugs or imaging agents in vivo [15].

Considering that numerous isomerases, epimerases and racemases exist, would it be possible that D-amino acids are converted in vivo to their L-enantiomers, which would then be susceptible to protease degradation? Such a fate would overall reduce the prolonged half-life of peptides bearing Damino acids. Actually, all eukaryotic peptide isomerases known to date do not process free amino acids, rather, they act on single residues of intact peptides and require specific flanking sequences for efficient catalysis [20]. Epimerases and racemases that act on single amino acids do exist, but their presence has been reported mainly in bacteria and some plants, effectively making this enzyme class an interesting drug target [21]. In addition, their natural substrates are usually L-amino acids, which are converted to D-, and the opposite reaction may occur but at a much slower rate [22]. Besides, although D-amino acids are increasingly being reported in human aged proteins and often related to aging diseases, their occurrence is typically explained by means of non-enzymatic racemization [23] with only a very few exceptions [24]. Therefore, although we cannot exclude the possibility of D-amino acid racemization in vivo, this option does not appear to be their most likely fate in the human body.

Nevertheless, the assumption that all enzymes, and not just proteases, will always process D-amino acids at a slower rate than their L-counterparts, if indeed at all, can be at times misleading. Care should be taken in this sense, especially in light of enzymatic promiscuity. In fact, promiscuous enzymes can process unnatural molecules, or molecules other than their natural substrates, or even catalyze different or opposite reactions than those for which they are designed by nature [25]. For instance, it was recently shown that a phosphatase dephosphorylates both D- and L-phosphotyrosine- containing peptides, and at the same rate. This finding can be exploited to control the rate of self-assembly, for instance by administering a non-assembling phosphate precursor that in situ will be enzymatically converted to a gelling peptide [13]. There are also other examples of promiscuous enzymes that can be exploited to synthesize heterochiral gelling peptides in environmentally-friendly ways, e.g. via lipasecatalyzed reverse hydrolysis [26]. Interestingly, D-peptide or D,L-heterochiral peptide synthesis was achieved with overall higher conversion yield, relative to the L-homochiral analogue. This can be explained if we consider that a dynamic equilibrium exists, where because of the enzyme's higher affinity for L-peptides, those will be preferentially hydrolyzed, and the equilibrium shifted against their production, while the opposite holds true for peptides containing Damino acids [26].

\section{EFFECTS ON PEPTIDE CONFORMATION}

\subsection{Beta-Turns and Beta-Hairpins}

The use of D-amino acids can be exploited for other reasons, such as their effects on peptide secondary structure conformation (Fig. 1). One of the earliest studies seeking identification of minimal peptide motifs adopting stable secondary structures dates back almost 20 years. From a survey of the protein database the authors designed a 23-mer capable of adopting a beta-hairpin conformation (i.e. two beta sheets connected by a beta turn, forming a "U" structure, Fig. 1A), whose stability was then improved through an iterative process [27]. The stabilizing effect of a single Dproline in a heterochiral type II' turn, as a central structural nucleation element, was a crucial finding. Importantly, neither the hairpin nor the tertiary structure were observed in the homochiral control peptide. This study was the first to highlight the key structural role played by a single D-amino acid, amongst over 20 L-amino acids, in a 23 -mer peptide secondary conformation.

Further developments by other authors eventually led to the use of a D-proline-based beta-turn in a 20-mer betahairpin motif, able to self-assemble in a macroscopic hydrogel [28]. This self-assembling 20-mer is relevant to medicinal application. It is compatible with fibroblast cell culture conditions and has shown a good performance in terms of cytotoxicity in vitro [29]. Interestingly, this amphiphilic peptide, rich in cationic lysine residues, also displays antibacterial properties (see sections below) [30].

From a medicinal perspective, beta-turn stabilization by substitution of L-amino acids with their D-enantiomers is now a well-known phenomenon that can be an effective tool to increase the binding affinity, and/or biological stability, of bioactive peptides [31]. For instance, D-phenylalanine can be used to this end to generate a potent, and long-lasting, analogue of $\alpha$-melanocyte-stimulating hormone [32].

\subsection{Beta-Sheets}

The introduction of D-amino acids can also have disruptive effects on peptide secondary conformation, depending on the amino acid sequence. Introduction of one Dphenylalanine residue in different positions of naphthalenebased dipeptide gelators was shown to hamper hydrogela- 
tion. The authors ascribed this fact to reduced intermolecular interactions, and thus reduced formation of superstructures, possibly due to an altered peptide configuration [19]. In another report, introduction of D-amino acids in different positions along a self-assembling peptide sequence led to disruption of the beta-sheet secondary conformation (Fig. 1B) and general loss of secondary structure suggesting that, at least for that sequence, homochirality is a requirement for peptide self-assembly [16].

Recent studies on unprotected tripeptides bearing the Phe-Phe motif and prone to forming beta-sheets revealed an opposite trend. In particular, some of us analyzed the supramolecular behavior of the complete Phe-Phe-Val stereoisomer series under physiological conditions [33]. This is one of the first, and shortest, unprotected peptide sequences identified as a gelator at neutral $\mathrm{pH}$ [34]. Circular dichroism data revealed a special role played by the central amino acid on the secondary conformation (Fig. 2). Interestingly, this residue alone dictates the chirality of the supramolecular arrangements, even for tripeptides with the other two amino acids in opposite stereoconfiguration. However, having a Damino acid in this position is not sufficient per se to achieve macroscopic hydrogelation for Phe-Phe-Val peptides [33]. Self-supportive hydrogels arising from stabilized supramolecular beta-sheet arrangements were observed for the Phe-Phe-Val stereoisomers bearing an $\mathrm{N}$-terminal amino acid with stereoconfiguration different from the other two amino acids (i.e. ${ }^{\mathrm{D}} \mathrm{Phe}-{ }^{\mathrm{L}} \mathrm{Phe}-{ }^{\mathrm{L}} \mathrm{Val}$ and its enantiomer ${ }^{\mathrm{L}} \mathrm{Phe}-{ }^{\mathrm{D}} \mathrm{Phe}-$ ${ }^{\mathrm{D}}$ Val). Limited beta-sheet nanostructures were observed by microscopy in the case of other heterochiral, but not homochiral, peptides, and importantly, both hydrogels with either L- or D-supramolecular chirality supported fibroblast cell culture and did not result in cell cytotoxicity in solution [33].

These are not the only reports of D-amino acids promoting beta-sheet formation. For instance, one study analyzed a series of peptides made of two $\gamma$-amino acids at the $N$ terminal, followed by the D-pentamer ${ }^{\mathrm{D}} \mathrm{Lys}-{ }^{\mathrm{D}} \mathrm{Leu}-{ }^{\mathrm{D}} \mathrm{Val}-{ }^{\mathrm{D}} \mathrm{Phe}-$ ${ }^{\mathrm{D}} \mathrm{Phe}$. Considering that $\gamma$-peptides are prone to helix formation, the D-pentamer, which is prone to beta-sheet formation, had a predominant effect on the overall peptide secondary conformation. In one example, the beta-sheet structure was observed in dilute solutions of concentrations at which other peptides do not fold, suggesting a stabilizing effect played by the amino acid sequence bearing the D-residues [35].

Finally, in one case an interesting temperature-dependent secondary conformation was observed only for a Denantiomer and not for its L-counterpart. The secondary conformation stability was tested by circular dichroism under various conditions of $\mathrm{pH}$, ionic strength, temperature, and denaturing agents (i.e. urea or SDS). The two enantiomers displayed identical behavior under all conditions except for the thermal treatment. In particular, the L-enantiomer maintained a highly stable beta-sheet conformation at temperatures as high as $110^{\circ} \mathrm{C}$, while its D-enantiomer underwent a reversible transition towards alpha-helix with heating, suggesting a lower thermal stability of the supramolecular Damino acid arrangements [15].

\subsection{Alpha-Helices}

In contrast to beta-sheet forming peptides, we could locate only one report on heterochiral self-assembled gels based on alpha helices (Fig. 1C). Insertion of even a single D-amino acid in a 14-mer amphipathic peptide completely abolished hydrogel formation, suggesting that alpha-helices were better preserved in homochiral, rather than heterochiral, peptide sequences [36].

\section{EFFECTS ON SELF-ASSEMBLY AND GELATION}

It is difficult to predict the self-assembly behavior of heterochiral peptides and peptide derivatives, and it has been shown that even the insertion of a single D-amino acid in a peptide sequence can significantly alter the peptide supramolecular behavior, and in different ways, depending on the sequence and the D-amino acid position.

Some of us were the first to report on the use of amino acid chirality as a tool to induce gelation of unprotected tripeptides, the shortest peptide motifs that gel in in vitro physiological conditions, without the use of organic solvents [34]. In particular, we observed that, under conditions at which the homochiral sequences Val-Phe-Phe, Phe-Phe-Val, or Leu-Phe-Phe do not gel, their heterochiral analogues bearing a D-amino acid at the $N$-terminal rapidly form selfsupportive hydrogels based on antiparallel beta-sheets [34, 37]. Interestingly, despite the very subtle differences in amino acid sequence, the three heterochiral peptides ${ }^{\mathrm{D}} \mathrm{Val}-$ Phe-Phe, ${ }^{\mathrm{D}}$ Phe-Phe-Val, and ${ }^{\mathrm{D}}$ Leu-Phe-Phe form different nanostructures, i.e., nanotapes, twisted fibers, and thick bundles, respectively [34, 37]. Molecular modelling and XRD studies point to a supramolecular stabilizing effect played by heterochirality. By contrast with the homochiral stereoisomers, presence of both D- and L- amino acids removes key steric clashes between side chains, and allows for the formation of peptide stacks that interdigitate via phenylalanine zippers into superstructures with hierarchical order [37]. As a result, gelation occurs as rapidly as within seconds, as shown in a video [37].

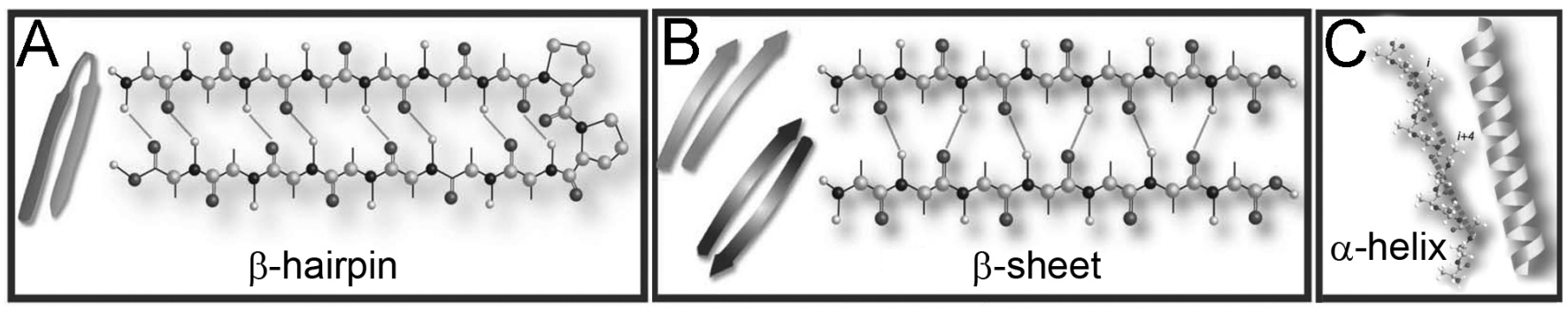

Fig. (1). Examples of peptide secondary conformation. Adapted from ref. [3] with kind permission from The Royal Society of Chemistry. 
A. CD spectra of enantiomers I

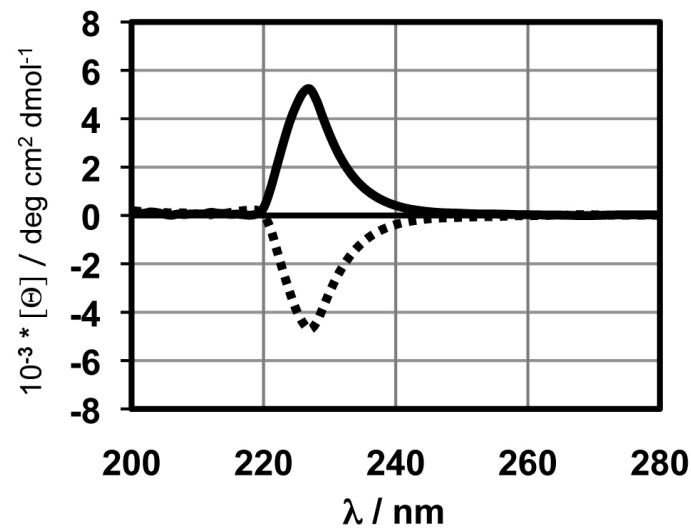

C. CD spectra of enantiomers III

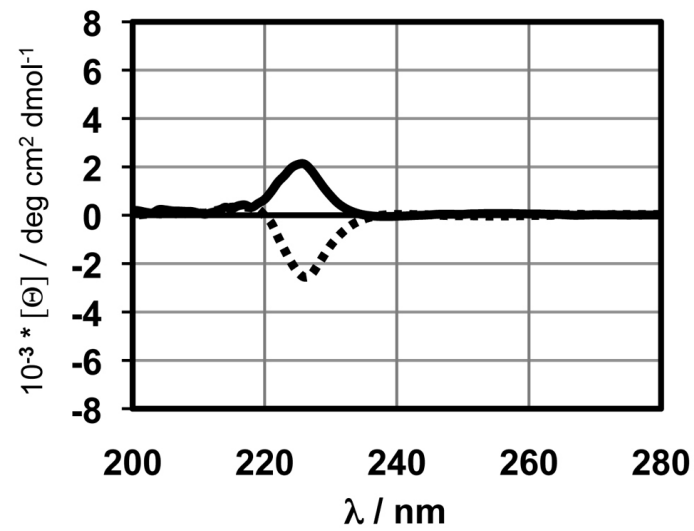

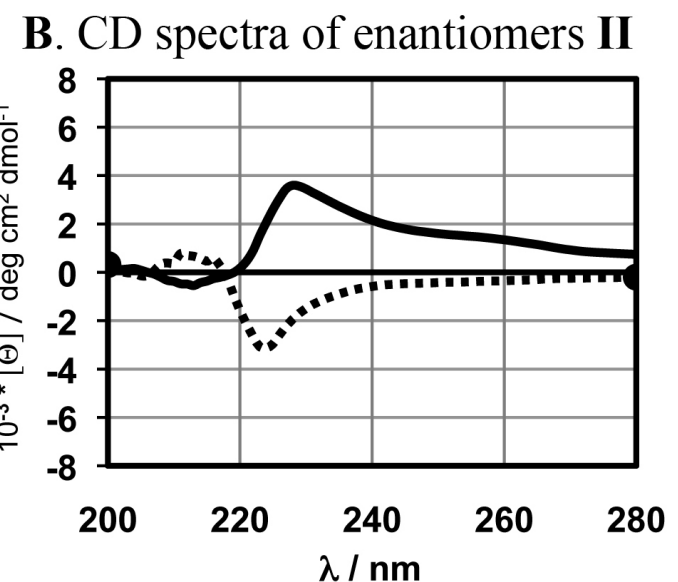

D. CD spectra of enantiomers IV

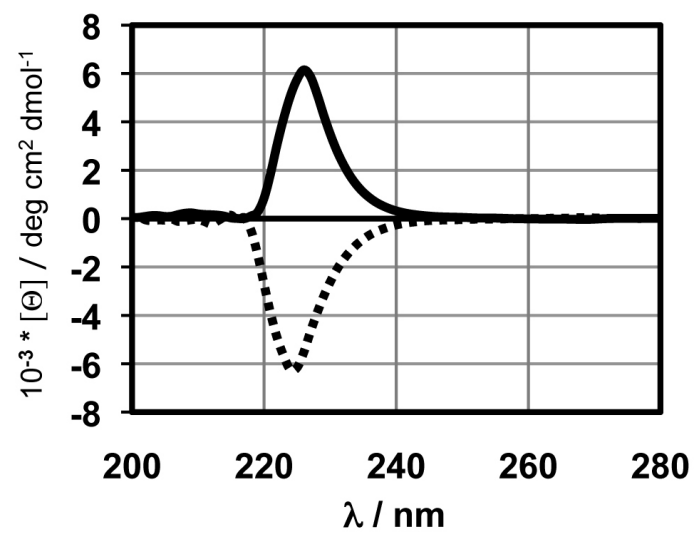

Fig. (2). Circular dichroism (CD) spectra of heterochiral Phe-Phe-Val tripeptides reveal that chirality of supramolecular arrangements is dictated by the stereoconfiguration of the central amino acid. A) ${ }^{\mathrm{L}} \mathrm{Phe}-{ }^{\mathrm{L}} \mathrm{Phe}-{ }^{\mathrm{L}} \mathrm{Val}$ (black line) and ${ }^{\mathrm{D}} \mathrm{Phe}-{ }^{\mathrm{D}} \mathrm{Phe}-{ }^{\mathrm{D}} \mathrm{Val}$ (dotted line) enantiomers; (B) ${ }^{\mathrm{D}} \mathrm{Phe}-{ }^{\mathrm{L}} \mathrm{Phe}-{ }^{\mathrm{L}} \mathrm{Val}$ (black line) and ${ }^{\mathrm{L}} \mathrm{Phe}-{ }^{\mathrm{D}} \mathrm{Phe}-{ }^{\mathrm{D}}$ Val (dotted line) enantiomers; (C) ${ }^{\mathrm{D}}$ Phe- ${ }^{\mathrm{L}} \mathrm{Phe}-{ }^{\mathrm{D}}$ Val (black line) and ${ }^{\mathrm{L}} \mathrm{Phe}-{ }^{\mathrm{D}} \mathrm{Phe}-{ }^{\mathrm{L}} \mathrm{V}$ al $($ dotted line) enantiomers; (D) ${ }^{\mathrm{L}} \mathrm{Phe}-{ }^{\mathrm{L}} \mathrm{Phe}-{ }^{\mathrm{D}} \mathrm{Val}$ (black line) and ${ }^{\mathrm{D}} \mathrm{Phe}-{ }^{\mathrm{D}} \mathrm{Phe}-{ }^{\mathrm{L}} \mathrm{Val}$ (dotted line) enantiomers. Reprinted from ref. [33] with kind permission of The Royal Society of Chemistry.

Another interesting study analyzed the supramolecular behavior of low-molecular-weight gelators based on dipeptide amphiphiles (Fig. 3). This work showed that the chirality of the $C$-terminal amino acid dictates the chiral orientation of the supramolecular helical nanoribbons, with on one hand the ${ }^{\mathrm{L}}$ Ala- ${ }^{\mathrm{D}}$ Ala derivative having the same handedness as the ${ }^{\mathrm{D}}$ Ala- ${ }^{\mathrm{D}}$ Ala counterpart, and on the other hand the ${ }^{\mathrm{D}}$ Ala- ${ }^{\mathrm{L}}$ Ala derivative having the same handedness as the ${ }^{\mathrm{L}}$ Ala- ${ }^{\mathrm{L}}$ Ala analogue [38].

In addition, homochiral and heterochiral effects have been analyzed for the case of multi-component selfassembled hydrogels, where homochirality of the peptide building blocks appeared to confer mechanical advantages relative to mixtures of both L- and D-peptides [39]. Contrarily, the opposite trend has been reported for racemic mixtures assembling into nanostructured gels. In one example of a beta-hairpin peptide an equimolar mixture of the two enantiomers displayed enhanced mechanical rigidity, with an elastic modulus $G$ ' that was four times higher than that of each peptide gel, under identical conditions. Microscopy images revealed an unaltered nanofibril morphology and the authors proposed that enantiomers may interact either at the molecular, or at the supramolecular, level forming mixed fibrils or self-segregating into separate fibrils, respectively (Fig. 4) [40].

Follow-up work by other authors on amphipathic peptides showed that enantiomers do not self-sort, rather they form hybrid beta-sheets with alternating L- and D-peptides, as confirmed by FRET analysis. The elegant study labelled the peptides with a fluorophore/quencher Förster pair with a radius of $3.3 \mathrm{~nm}$. Fluorescence endured upon mixing of preformed fibrils of each peptide enantiomer, while pre-mixing of an equimolar racemic mixture displayed complete fluorescence quenching, indicating that the two enantiomers were homogeneously distributed within a distance of $3.3 \mathrm{~nm}$ between each other, as would occur in alternating positions within beta-sheets [41].

Interestingly, an earlier study on enantiomers of polylysine reported that the equimolar racemate formed selfassembled amyloid-like nanofibrillar structures arising from beta-sheets at room temperature, while each enantiomer singly required higher temperatures to undergo an alpha helixto beta-sheet transition. The authors postulated a stabilized supramolecular structure in the former [42]. 

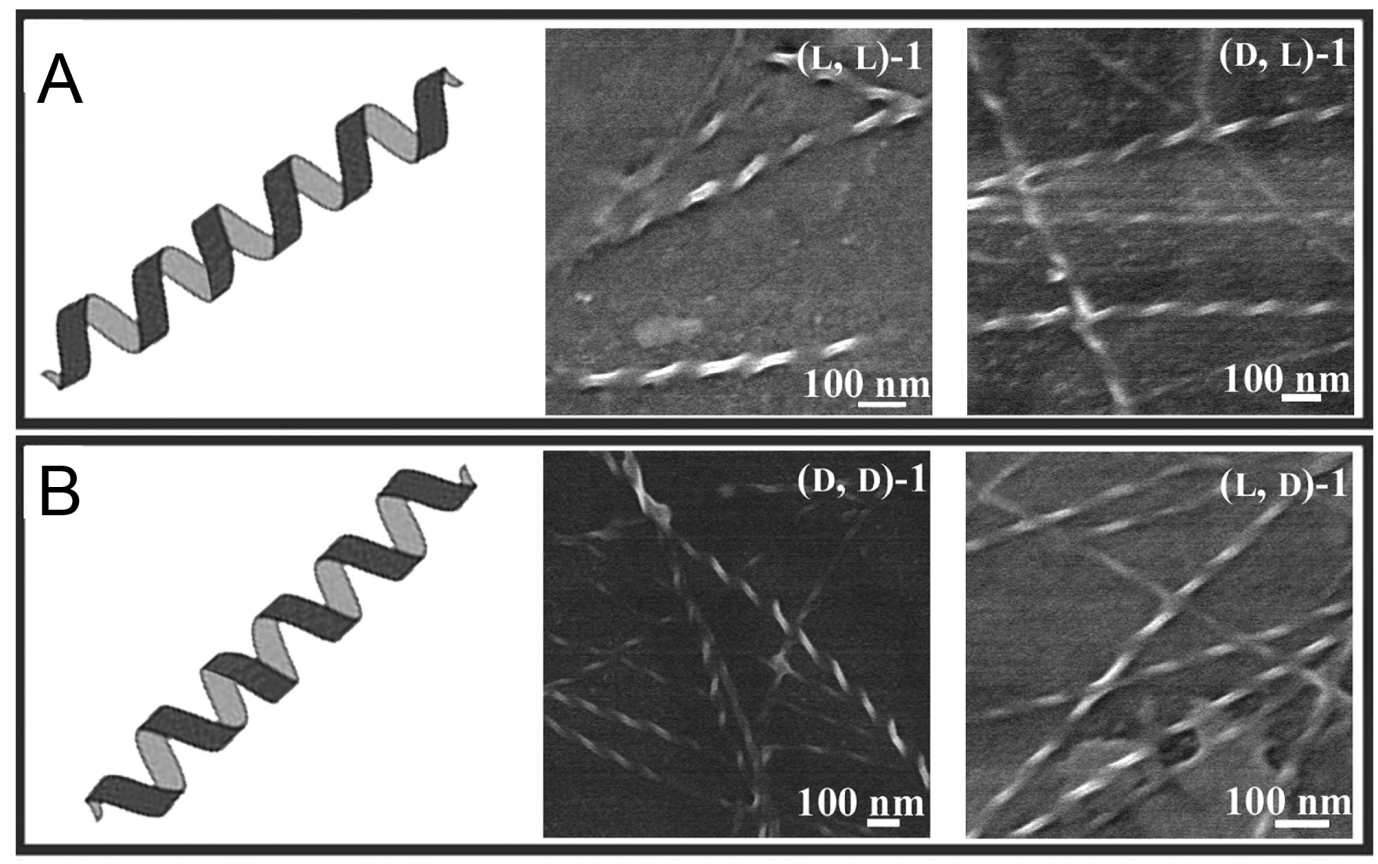

Fig. (3). Ala-Ala dipeptide amphiphiles form helical nanoribbons with a handedness dictated by chirality of the $C$-terminal amino acid, as observed by AFM. A. left-handed nanoribbons. B. right-handed nanoribbons. Adapted with permission from ref. [38]. Copyright (C) 2013 American Chemical Society.
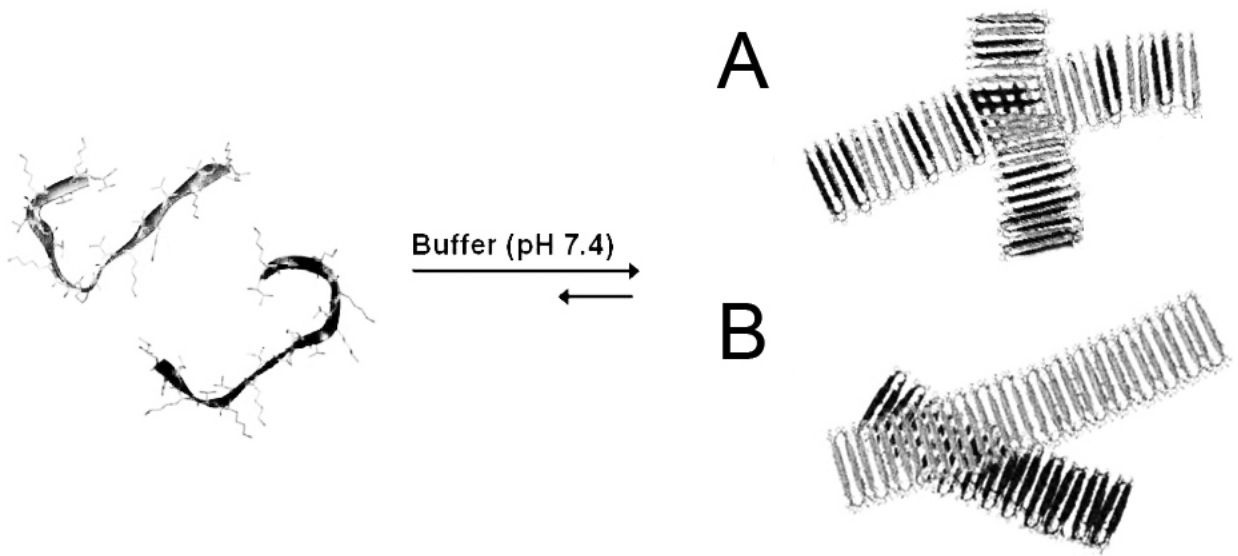

Fig. (4). An equimolar mixture of two self-assembling peptide enantiomers can give rise to A. mixed fibrils, each containing both peptides, or B. self-sorted fibrils, each containing one peptide only. Adapted with permission from ref. [40]. Copyright (C) 2011 American Chemical Society.

Overall, all these studies are in agreement with a stabilized supramolecular amyloid-like beta-sheet nanostructure arising from the equimolar mixture of two peptide enantiomers, relative to each peptide alone. This is an intriguing finding that deserves further investigation as it may have important implications for the design of future lead candidates to treat amyloidosis.

\section{THERAPEUTIC EFFECTS}

\subsection{Antimicrobial Activity}

Heterochiral gelling peptides have also shown interesting antibacterial properties. In particular, a 20-mer beta-hairpin peptide hydrogel displayed antibacterial activity exerted through membrane disruption [30]. In light of its polyca- tionic nature it is possible that its mechanism of action is analogous to the one of antimicrobial peptides carrying positive charges [43]. In this regard it is interesting to note that the introduction of D-amino acids can also enhance the activity and selectivity of antimicrobial peptides [44]. When the side chains are not ionizable the situation is different. For instance, some of us reported on the use of the heterochiral gelling ${ }^{\mathrm{D}}$ Leu- ${ }^{\mathrm{L}} \mathrm{Phe}-{ }^{\mathrm{L}} \mathrm{Phe}$ tripeptide as biomaterial, which surprisingly displayed a mild antibacterial activity against the Gram-negative strains E.coli and a clinical strain of K.pneumoniae (Fig. 5) [45]. Interestingly, such an activity was observed neither in the self-assembling gelling analogue ${ }^{\mathrm{D}}$ Val- ${ }^{\mathrm{L}} \mathrm{Phe}-{ }^{\mathrm{L}} \mathrm{Phe}$, which differs by only a methylene unit, nor in the case of the scrambled sequence ${ }^{\mathrm{L}} \mathrm{Phe}-{ }^{\mathrm{D}} \mathrm{Leu}-{ }^{\mathrm{L}} \mathrm{Phe}$ or of single amino acids ${ }^{\mathrm{D}}$ Leu and ${ }^{\mathrm{L}} \mathrm{Phe}$. This observation suggests the effect is sequence-dependent and not simply due to the 


\section{S. aureus}

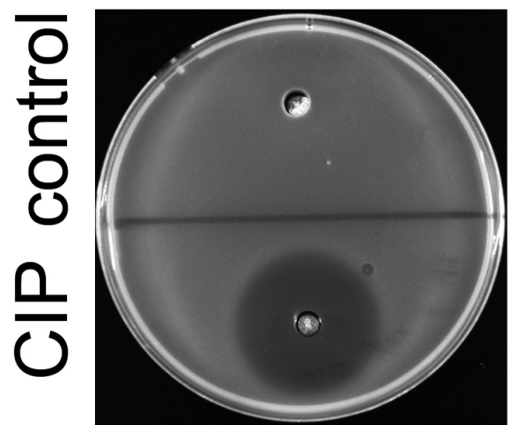

E.coli

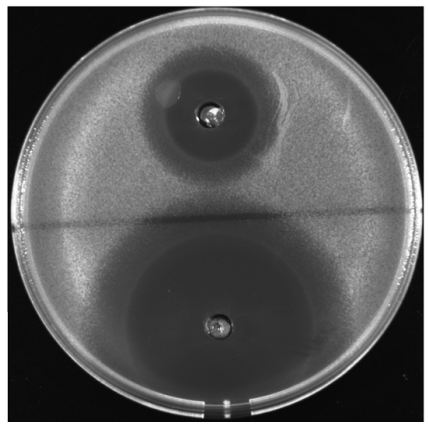

K.pneumoniae

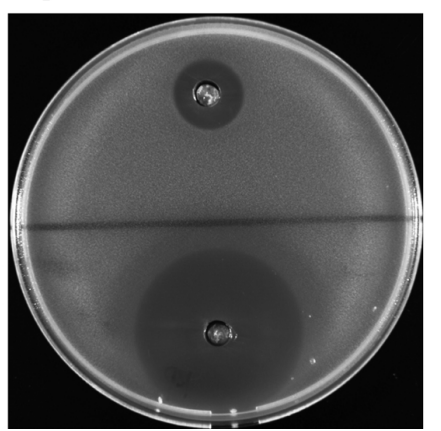

Fig. (5). Antibacterial activity of self-assembling ${ }^{\mathrm{D}} \mathrm{Leu}-{ }^{\mathrm{L}} \mathrm{Phe}-{ }^{\mathrm{L}} \mathrm{Phe}$ peptide gel alone (i.e., control, top half of agar plates) or co-assembled with antibiotic ciprofloxacin (i.e., CIP, bottom half of agar plates). Reprinted from ref. [45] Copyright (C) 2013 with permission from Elsevier.

supramolecular arrangement, giving scope for the development of novel antimicrobial peptides. Importantly, no hemolytic side effect was observed despite this being an often observed adverse phenomenon for antimicrobial peptides. Nevertheless, further studies are needed to elucidate the exact mechanism of action and further investigate the possibility to optimize the antibacterial activity.

\subsection{Amyloidosis Therapy}

The rationale to use self-assembling peptides bearing Damino acids to treat amyloidosis comes from the fact that many self-assembling peptide sequences are indeed inspired from the core residues found in various amyloids, with Alzheimer's $A \beta(1-42)$ peptide being the most studied [46]. Specifically, a number of variations around the Lys-Leu-ValPhe-Phe pentamer of $A \beta$ have been designed to achieve soft materials, and even the pentamer itself displayed the ability to form hydrogels [47]. In light of the tendency of the peptide to bind to itself, thus forming the so-called amyloid aggregates, it can be envisaged that small peptides of similar amino acid sequence may compete with such binding, thus interfering with amyloid formation. As far as D-amino acids are concerned, it is worth noting that $N$-terminal conjugation of ${ }^{\mathrm{D}}$ Lys- ${ }^{\mathrm{D}}$ Leu- $-{ }^{\mathrm{D}}$ Val- ${ }^{\mathrm{D}} \mathrm{Phe}-{ }^{\mathrm{D}} \mathrm{Phe}$ with a $\gamma$-dipeptide generated a promising lead compound to treat amyloidosis (Fig. 6). The resulting heptamer was capable of binding to the $A \beta(1-42)$ peptide, thus reducing its cytotoxicity with a favourable dose-response curve at physiologically relevant concentration conditions [35].

\subsection{Enhancement of Drug Selectivity}

The covalent appendage of a peptide gelator to a drug can offer interesting means to fine-tune drug activity and selectivity. For instance, the anti-inflammatory drug naproxen displays a convenient carboxylic acid group that can be used to couple a peptide via either a terminal amine or via the amino side chain of a lysine residue [48]. Molecular modelling studies of the COX-2 target enzyme showed that the active site could accommodate a rather bulky ${ }^{\mathrm{D}} \mathrm{Phe}-{ }^{\mathrm{D}} \mathrm{Phe}$ dipeptide without compromising drug activity. In fact, experimental $\mathrm{IC}_{50} \mathrm{~S}$ of drug conjugates with short, gelling, Dpeptides showed increased values relative to the drug alone. However, in the case of ${ }^{\mathrm{D}} \mathrm{Phe}-{ }^{\mathrm{D}} \mathrm{Phe}-{ }^{\mathrm{D}}$ Lys- ${ }^{\mathrm{D}}$ Tyr the increase was only minor and did not inhibit drug activity. Importantly, COX-2 selectivity over COX-1 appeared improved for the drug-peptide conjugates relative to the drug alone, and the effect was not observed in the case of the L-peptide enantiomers. In principle this approach offers an elegant method to enhance drug selectivity and reduce COX-1 dependent side effects [48].

\subsection{Hemostasis}

Gel formation has also been envisaged as a means to create an artificial blood clot to treat hemorrhages in fibrinogendeficient patients. The authors designed a compound featuring a PEG-D-peptide conjugate to assist with solubility, followed by a thrombin-recognition motif, and a D-peptide gelator (Fig 7). Despite the complexity of human plasma composition, addition of the compound and thromboplastin to fibrinogen-depleted human plasma led to a cascade of events, namely: 1) thrombin activation by thromboplastin, 2) compound enzymatic cleavage by activated thrombin, and 3 ) gelation, effectively forming an artificial blood clot. The authors envisaged use of the system in bandages to treat severe wounds and traumas [42].

Another study exploited gelation of an amphipathic Dpeptide to stop in vivo bleeding arising from a liver wound in a rabbit model. The peptide and its enantiomer, thanks to their self-assembling ability, were able to lead to hemostasis within 20 seconds. In contrast, the D,L-heterochiral analogues, which presented minor self-assembling ability, took significantly longer to have the same effect [13]. The process exploited the gelation-promoting role played by the abundant salts naturally present in blood to accelerate self-assembly.

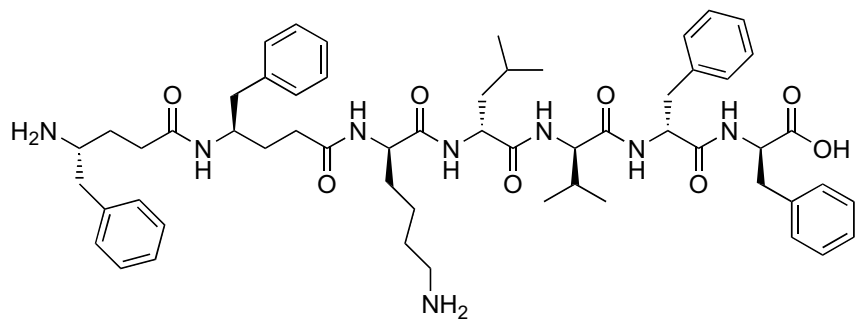

Fig. (6). Promising lead candidate to inhibit $A \beta$ peptide aggregation [35]. 


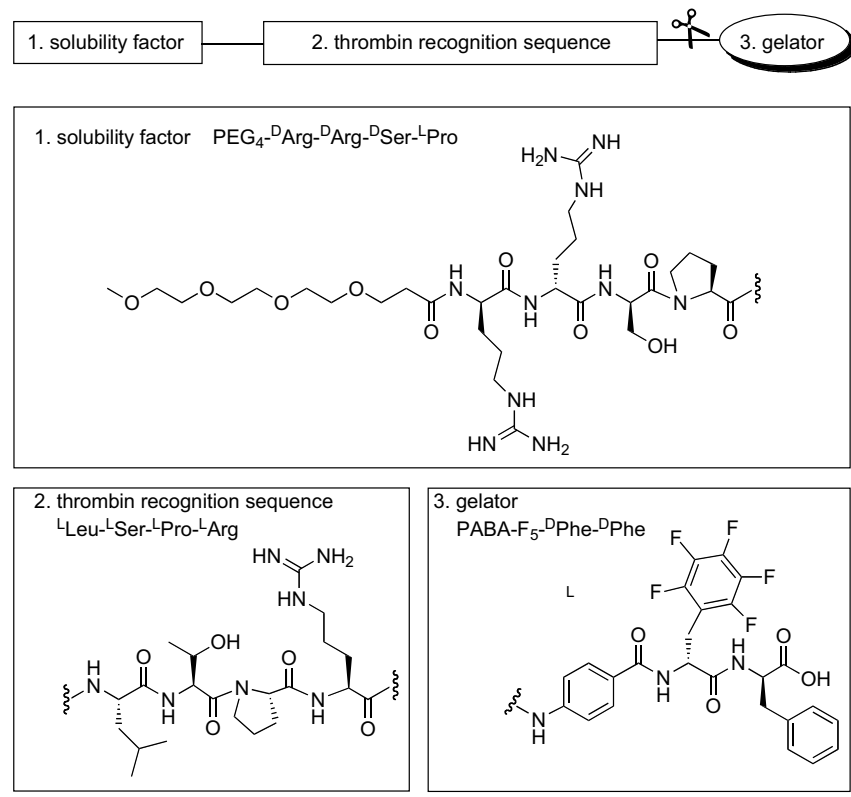

Fig. (7). Heterochiral peptide derivative with a thrombin cleavage site (indicated by the scissors) to release a self-assembling gelator [42].

\section{DRUG DELIVERY APPLICATIONS}

Hydrogels are widely used as vehicles for drug delivery, and so it is not surprising that systems of gelling peptides bearing D-amino acid(s) were designed for this purpose. Drug loading and delivery can be achieved in different ways. From a purely chemical point of view we can envisage the drug being bound either non-covalently or covalently to the gel matrix, and examples of both will be briefly discussed below.

The most obvious non-covalent approach consists of simple drug encapsulation into the hydrogel matrix. For instance, curcumin could be used as a model compound for hydrophobic drugs. The drug could be dissolved with various amounts of the peptide gelator in order to fine-tune the gel's viscoelastic properties, and thus the drug release rate [49]. It is worth noting that this is simply a physical process, since the circular dichroism spectroscopic profile of the gel was not altered by the presence of the drug, suggesting that the peptide assembled in the same manner either with or without curcumin [49]. A similar approach can be applied also to other hydrophobic drugs. 10-hydroxycamptothecin was encapsulated into D-amino acid bearing self-assembling peptides without altering the fiber nanomorphology. It is worth noting that the heterochiral peptide was able to increase the aqueous stability of the drug to a higher extent than the L-homochiral peptide counterpart, suggesting a potential use as stabilizer in drug formulations [6].

A more interesting option offered by supramolecular gels is the possibility of the drug to participate in the assembly process, i.e. being integrated in the gel matrix architecture. For instance, a poorly soluble hydrophobic drug can interact with the hydrophobic heterochiral tripeptide ${ }^{\mathrm{D}}$ Leu-Phe-Phe via $\pi-\pi$ stacking, as shown by circular dichroism (CD) spectroscopy [45]. Co-assembly clearly affected the supramolecular architecture, yielding different nanomorphol- ogy and different rheological properties of the resulting soft material. Importantly, thanks to the competition between drug-peptide and drug-drug interactions, precipitation did not occur and the drug could be loaded in the hydrogel at physiological conditions, and at concentrations superior to its solubility limit [45]. Clearly this approach offers an interesting alternative for the delivery of poorly-soluble drugs.

Alternatively, gelling peptides and drugs can be covalently linked to each other. As an example, the carboxylic end of the anti-inflammatory drug naproxen was bound to the D-peptides ${ }^{\mathrm{D}}$ Phe- ${ }^{\mathrm{D}}$ Phe- ${ }^{\mathrm{D}}$ Lys, ${ }^{\mathrm{D}} \mathrm{Phe}-{ }^{\mathrm{D}} \mathrm{Phe}-{ }^{\mathrm{D}} \mathrm{Tyr}$, and ${ }^{\mathrm{D}} \mathrm{Phe}-$ ${ }^{\mathrm{D}}$ Phe- ${ }^{\mathrm{D}}$ Lys- ${ }^{\mathrm{D}}$ Tyr. Hydrogels were obtained at neutral $\mathrm{pH}$, either by using a combined temperature and $\mathrm{pH}$ trigger, or by using phosphotyrosine-bearing non-gelling precursors that were dephosphorylated in situ upon action of a phosphatase [48]. The covalent conjugation of the drug with a D-peptide to obtain a hydrogel for medicinal use is not a trivial matter and can have dramatic effects on the gelation properties. Indeed, conjugation of the short sequence ${ }^{\mathrm{D}} \mathrm{Phe}-{ }^{\mathrm{D}} \mathrm{Phe}$ hampered the gelation process at neutral $\mathrm{pH}$, and formed a compound that gelled only at a $\mathrm{pH}$ as acidic as 4 [48].

Finally, one study showed that peptides containing Damino acids, when self-assembled, enriched at the cell membrane, but not when concentrations were too low for the supramolecular structures to arise. This observation could in principle be exploited for the targeted delivery of a small molecule that has a specific site of action located at the cell membrane [50].

\section{BIOMATERIALS USE AND CYTOTOXICITY}

Self-assembled peptides are popular building blocks for soft biomaterials thanks to their well-established chemistry, natural occurrence, possibility to encode a biological message, and biodegradability [51]. Besides, nanostructured peptide hydrogels offer an ideal environment for tissue regeneration and wound healing, by providing a good mimic of the physicochemical properties of the extracellular matrix as well as maintaining an appropriate level of moisture to favor cellular metabolic exchanges and movement [5].

However, in the case of heterochiral peptides or Dpeptides not much is known about their biocompatibility, and in fact only a few reports have addressed the issue thus far. One may presume that in light of their lower enzyme sensitivity relative to L-peptides, D-peptides may lead to more adverse effects in vitro, and perhaps more so in vivo. Indeed, there is one report of a L-peptide gel leading to milder inflammation response relative to the D-enantiomer in vivo, with a thicker collagen capsule being formed around the latter [18]. Nevertheless, cytotoxicity has to be ascertained case by case.

In terms of comparisons between enantiomers, obviously biological effects will be sequence-dependent, just as in the case of chiral small molecules, where stereochemistrydependent biological effects of each enantiomer are strongly dependent on the small molecule chemical identity. In the case of homochiral peptides, at times it is the D-peptide derivative that is less cytotoxic in cell culture in vitro than its L-analogue [52]. On the other hand, other examples were reported of the L-enantiomer in vitro having lower cytotoxic- 


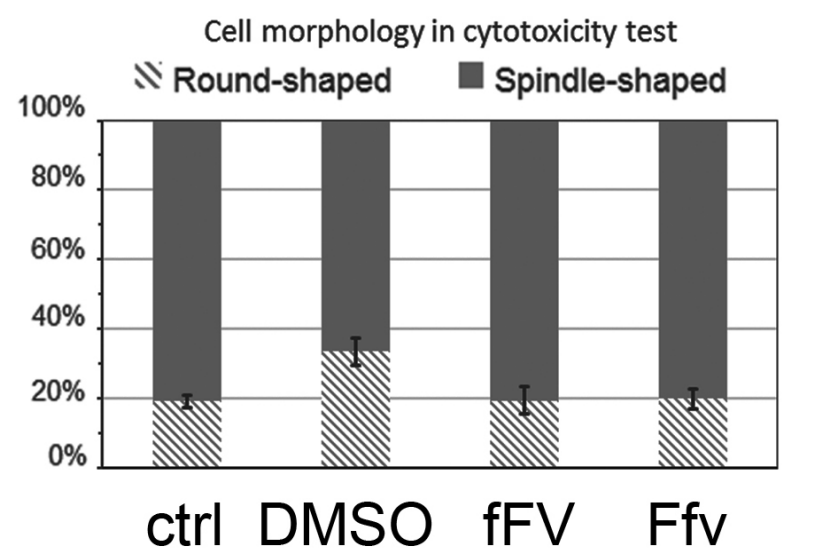

\section{Cell culture on hydrogels at $48 \mathrm{~h}$}

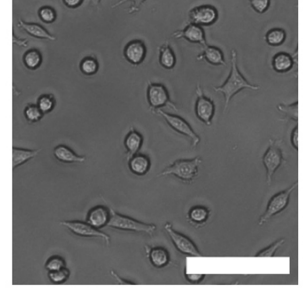

ctrl

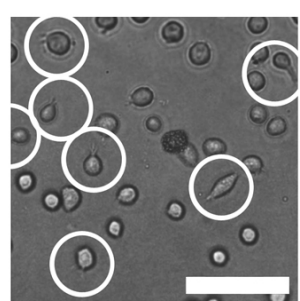

fFV gel

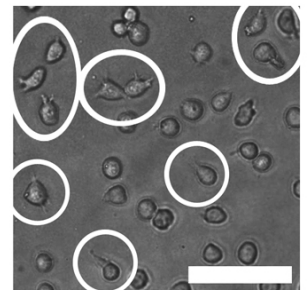

Ffv gel

Fig. (8). Heterochiral self-assembling peptides ${ }^{\mathrm{D}} \mathrm{Phe}-{ }^{\mathrm{L}} \mathrm{Phe}-{ }^{\mathrm{L}}$ Val (i.e., fFV) and ${ }^{\mathrm{L}} \mathrm{Phe}-{ }^{\mathrm{D}} \mathrm{Phe}-{ }^{\mathrm{D}}$ Val (i.e., Ffv) perform equally well in cytotoxicity tests both in solution (left) and as gels (right). Circles indicate spreading cells. Scale bar $=50 \mu \mathrm{m}$. Adapted from ref. [33] with kind permission of The Royal Society of Chemistry.

ity than either its D-counterpart or the racemic mixture [53]. Finally, there are also cases of identical performance for either enantiomer used as 3D-scaffold for cell-culture cytotoxicity assays in vitro [54].

Similarly, and also in the case of heterochiral peptide gels, it is difficult to anticipate which enantiomer will perform better form a biological viewpoint. As mentioned in the sections above, some of us identified the first uncapped tripeptides capable of gelation at physiological conditions, without the aid of organic solvents, and indeed these compounds were envisaged for their use as nanostructured biomaterials [55]. These hydrophobic, heterochiral peptides performed well in fibroblast cell culture, even in the case of supramolecular structures with unnatural D-chirality (Fig. 8). Interestingly, cell penetration within the gel, and instances of cell spreading, were observed despite the absence of bioactive motifs promoting cell adhesion. No obvious difference between the biological effects induced by each heterochiral enantiomer was noted [33]. This data suggested an overall benign interaction with cells, as supported by cytotoxicity assays on the peptides in solution [33], and by hemolytic assays [45]. Also other authors reported lack of cytotoxicity for heterochiral self-assembling peptides. Use of selfassembling heterochiral peptides bearing the tumor targeting RGD-sequence, a motif binding to integrin proteins [56], led to good tumor selectivity both in vivo and in vitro, with lower toxicity observed for non-cancerous cells [6].

\section{CONCLUSION}

Introduction of D-amino acids to self-assembling peptide gels started quite a few years ago with the simple aim to achieve higher stability against enzymatic degradation, and to thus enhance their durability as biomaterials. However, as more and more investigators adopted the use of D-amino acids in hydrogel biomaterials, unexpected effects on peptide secondary conformation, self-assembly behavior, and even biological performance were observed. Overall, this growing body of work is showing that peptide chirality has a lot more to offer than simply enzyme stability, and often hints at benefits that might be exploited by the medicinal chemist. Enhancements in drug delivery, selectivity, and potency have been achieved through the use of peptides displaying Damino acid(s) in their sequence. Novel paradigms of therapy and cell compartment targeting are being envisaged too. We believe that peptide chirality is indeed a versatile tool for the medicinal chemist, and creativity is the only limit for its wider application to find novel solutions in therapy for the $21^{\text {st }}$ century.

\section{LIST OF ABBREVIATIONS}

$$
\begin{aligned}
& \text { Ala }=\text { alanine } \\
& \text { Arg }=\text { arginine } \\
& \mathrm{CD}=\text { circular dichroism } \\
& \text { COX }=\text { cyclo-oxygenase } \\
& \text { FRET }=\text { Förster Resonance Energy Transfer } \\
& \text { Leu }=\text { leucine } \\
& \text { Lys }=\text { lysine } \\
& \text { Phe }=\text { phenylalanine } \\
& \text { Pro }=\text { proline } \\
& \text { RGD }=\text { arginine-glycine-aspartic acid } \\
& \text { SDS }=\text { Sodium Dodecyl Sulphate } \\
& \text { Ser }=\text { serine } \\
& \text { Thr }=\text { threonine } \\
& \text { Tyr }=\text { tyrosine } \\
& \text { Val }=\text { valine } \\
& \text { XRD }=\text { X-Ray Diffraction }
\end{aligned}
$$

\section{CONFLICT OF INTEREST}

The author(s) confirm that this article content has no conflict of interest.

\section{ACKNOWLEDGEMENTS}

SM is grateful to the Italian Ministry of Education, University and Research (MIUR) SIR program for funding under grant agreement $\mathrm{n}$. RBSI14A7PL, HOT-SPOT project. 


\section{REFERENCES}

[1] Marchesan, S.; Prato, M. Nanomaterials for (nano)medicine. ACS Med. Chem. Lett., 2013, 4 (2), 147-149.

[2] Rawat, A.; Nagaraj, R. Peptide self-assembly: From toxins to amyloid fibrils and nanotubes. Curr. Top. Med. Chem., 2014, 14 (6), 740-746; Gazit, E. Self-assembled peptide nanostructures: The design of molecular building blocks and their technological utilization. Chem. Soc. Rev., 2007, 36 (8), 1263-1269.

[3] Dasgupta, A.; Mondal, J. H.; Das, D. Peptide hydrogels. RSC Adv., 2013, 3 (24), 9117-9149.

[4] Lowik, D. W. P. M.; Leunissen, E. H. P.; van den Heuvel, M.; Hansen, M. B.; van Hest, J. C. M. Stimulus responsive peptide based materials. Chem. Soc. Rev., 2010, 39 (9), 3394-3412.

[5] Luo, Z.; Zhang, S. Designer nanomaterials using chiral selfassembling peptide systems and their emerging benefit for society. Chem. Soc. Rev., 2012, 41 (13), 4736-4754.

[6] Liu, J.; Liu, J.; Chu, L.; Zhang, Y.; Xu, H.; Kong, D.; Yang, Z.; Yang, C.; Ding, D. Self-assembling peptide of d-amino acids boosts selectivity and antitumor efficacy of 10hydroxycamptothecin. ACS Appl. Mater. Interfaces, 2014, 6 (8), 5558-5565.

[7] Adams, D. J. Dipeptide and tripeptide conjugates as lowmolecular-weight hydrogelators. Macromol. Biosci., 2011, 11 (2), 160-173.

[8] Marchesan, S. Recent patents on peptide self-assembled hydrogels as nanostructured biomaterials. Recent Pat. Nanomed., 2014, 4 (2), 77-81.

[9] Turner, R. D.; Vollmer, W.; Foster, S. J. Different walls for rods and balls: The diversity of peptidoglycan. Mol. Microbiol., 2014, $91(5), 862-874$

[10] Wolosker, H.; Blackshaw, S.; Snyder, S. H. Serine racemase: A glial enzyme synthesizing d-serine to regulate glutamate-n-methyld-aspartate neurotransmission. Proc. Natl. Acad. Sci. U.S.A., 1999, 96 (23), 13409-13414; Kim, P. M.; Duan, X.; Huang, A. S.; Liu, C. Y.; Ming, G.-1.; Song, H.; Snyder, S. H. Aspartate racemase, generating neuronal d-aspartate, regulates adult neurogenesis. Proc. Natl. Acad. Sci. U.S.A., 2010, 107 (7), 3175-3179.

[11] Lai, Y.; Xie, C.; Zhang, Z.; Lu, W.; Ding, J. Design and synthesis of a potent peptide containing both specific and non-specific celladhesion motifs. Biomaterials, 2010, 31 (18), 4809-4817.

[12] Wang, X.; Yan, C.; Ye, K.; He, Y.; Li, Z.; Ding, J. Effect of rgd nanospacing on differentiation of stem cells. Biomaterials, 2013, 34 (12), 2865-2874.

[13] Li, J.; Gao, Y.; Kuang, Y.; Shi, J.; Du, X.; Zhou, J.; Wang, H.; Yang, Z.; Xu, B. Dephosphorylation of D-peptide derivatives to form biofunctional, supramolecular nanofibers/hydrogels and their potential applications for intracellular imaging and intratumoral chemotherapy. J. Am. Chem. Soc., 2013, 135 (26), 9907-9914.

[14] Tugyi, R.; Uray, K.; Ivan, D.; Fellinger, E.; Perkins, A.; Hudecz, F. Partial D-amino acid substitution: Improved enzymatic stability and preserved ab recognition of a muc2 epitope peptide. Proc. Natl. Acad. Sci. U. S. A., 2005, 102 (2), 413-418; Sela, M.; Zisman, E. Different roles of D-amino acids in immune phenomena. FASEB J., 1997, $11(6), 449-456$

[15] Luo, Z.; Zhao, X.; Zhang, S. Structural dynamic of a selfassembling peptide $\mathrm{D}-\mathrm{EAK}_{16}$ made of only D-amino acids. PLoS ONE, 2008, 3 (5), e2364.

[16] Luo, Z.; Wang, S.; Zhang, S. Fabrication of self-assembling Dform peptide nanofiber scaffold $\mathrm{D}-\mathrm{EAK}_{16}$ for rapid hemostasis. Biomaterials, 2011, 32 (8), 2013-2020.

[17] Liang, G.; Yang, Z.; Zhang, R.; Li, L.; Fan, Y.; Kuang, Y.; Gao, Y.; Wang, T.; Lu, W. W.; Xu, B. Supramolecular hydrogel of a Damino acid dipeptide for controlled drug release in vivo. Langmuir, 2009, 25 (15), 8419-8422.

[18] Kang, E. Y.; Yeon, B.; Moon, H. J.; Jeong, B. PEG-L-paf and PEG-D-paf: Comparative study on thermogellation and biodegradation. Macromol., 2012, 45 (4), 2007-2013.

[19] Li, X.; Du, X.; Li, J.; Gao, Y.; Pan, Y.; Shi, J.; Zhou, N.; Xu, B. Introducing $\mathrm{D}$-amino acid or simple glycoside into small peptides to enable supramolecular hydrogelators to resist proteolysis. Langmuir, 2012, 28 (37), 13512-13517.

[20] Ollivaux, C.; Soyez, D.; Toullec, J. Y. Biogenesis of D-amino acid containing peptides/proteins: Where, when and how? J. Pept. Sci., 2014, 20 (8), 595-612.
[21] Conti, P.; Tamborini, L.; Pinto, A.; Blondel, A.; Minoprio, P.; Mozzarelli, A.; De Micheli, C. Drug discovery targeting amino acid racemases. Chem. Rev., 2011, 111 (11), 6919-6946.

[22] Bansal, P. S.; Torres, A. M.; Crossett, B.; Wong, K. K.; Koh, J. M.; Geraghty, D. P.; Vandenberg, J. I.; Kuchel, P. W. Substrate specificity of platypus venom L-to-D-peptide isomerase. J. Biol. Chem., 2008, 283 (14), 8969-8975.

[23] Ritz-Timme, S.; Collins, M. J. Racemization of aspartic acid in human proteins. Ageing Res. Rev., 2002, 1 (1), 43-59.

[24] Canu, N.; Ciotti, M. T.; Pollegioni, L. Serine racemase: A key player in apoptosis and necrosis. Front. Synapt. Neurosci., 2014, 6, 9.

[25] Tawfik, O. K.; S., D. Enzyme promiscuity: A mechanistic and evolutionary perspective. Annu. Rev. Biochem., 2010, 79 (1), 471505.

[26] Chronopoulou, L.; Sennato, S.; Bordi, F.; Giannella, D.; Di Nitto, A.; Barbetta, A.; Dentini, M.; Togna, A. R.; Togna, G. I.; Moschini, S. Designing unconventional fmoc-peptide-based biomaterials: Structure and related properties. Soft matter, 2014, 10 (12), 1944-1952.

[27] Struthers, M. D.; Cheng, R. P.; Imperiali, B. Design of a monomeric 23-residue polypeptide with defined tertiary structure. Science, 1996, 271 (5247), 342-345.

[28] Schneider, J. P.; Pochan, D. J.; Ozbas, B.; Rajagopal, K.; Pakstis, L.; Kretsinger, J. Responsive hydrogels from the intramolecular folding and self-assembly of a designed peptide. J. Am. Chem. Soc. 2002, 124 (50), 15030-15037.

[29] Kretsinger, J. K.; Haines, L. A.; Ozbas, B.; Pochan, D. J.; Schneider, J. P. Cytocompatibility of self-assembled $\beta$-hairpin peptide hydrogel surfaces. Biomaterials, 2005, 26 (25), 5177-5186.

[30] Salick, D. A.; Kretsinger, J. K.; Pochan, D. J.; Schneider, J. P. Inherent antibacterial activity of a peptide-based $\beta$-hairpin hydrogel. J. Am. Chem. Soc., 2007, 129 (47), 14793-14799.

[31] Hruby, V. J. Designing peptide receptor agonists and antagonists. Nat. Rev. Drug Discov., 2002, 1 (11), 847-858.

[32] Sawyer, T. K.; Sanfilippo, P. J.; Hruby, V. J.; Engel, M. H.; Heward, C. B.; Burnett, J. B.; Hadley, M. E., 4-norleucine, 7-Dphenylalanine-alpha-melanocyte-stimulating hormone: A highly potent alpha-melanotropin with ultralong biological activity. Proc. Natl. Acad. Sci. U. S. A., 1980, 77 (10), 5754-5758.

[33] Marchesan, S.; Easton, C. D.; Styan, K. E.; Waddington, L. J.; Kushkaki, F.; Goodall, L.; McLean, K. M.; Forsythe, J. S.; Hartley, P. G. Chirality effects at each amino acid position on tripeptide self-assembly into hydrogel biomaterials. Nanoscale, 2014, 6 (10), 5172-5180.

[34] Marchesan, S.; Easton, C. D.; Kushkaki, F.; Waddington, L.; Hartley, P. G. Tripeptide self-assembled hydrogels: Unexpected twists of chirality. Chem. Commun., 2012, 48 (16), 2195-2197.

[35] Castelletto, V.; Cheng, G.; Hamley, I. W. Amyloid peptides incorporating a core sequence from the amyloid beta peptide and gamma amino acids: Relating bioactivity to self-assembly. Chem. Commun., 2011, 47 (46), 12470-12472.

[36] Wieduwild, R.; Tsurkan, M.; Chwalek, K.; Murawala, P.; Nowak, M.; Freudenberg, U.; Neinhuis, C.; Werner, C.; Zhang, Y. Minimal peptide motif for non-covalent peptide-heparin hydrogels. J. Am. Chem. Soc., 2013, 135 (8), 2919-2922.

[37] Marchesan, S.; Waddington, L.; Easton, C. D.; Winkler, D. A.; Goodall, L.; Forsythe, J.; Hartley, P. G. Unzipping the role of chirality in nanoscale self-assembly of tripeptide hydrogels. Nanoscale, 2012, 4 (21), 6752-6760.

[38] Fu, Y.; Li, B.; Huang, Z.; Li, Y.; Yang, Y. Terminal is important for the helicity of the self-assemblies of dipeptides derived from alanine. Langmuir, 2013, 29 (20), 6013-6017.

[39] Taraban, M. B.; Feng, Y.; Hammouda, B.; Hyland, L. L.; Yu, Y. B. Chirality-mediated mechanical and structural properties of oligopeptide hydrogels. Chem. Mater., 2012, 24 (12), 2299-2310.

[40] Nagy, K. J.; Giano, M. C.; Jin, A.; Pochan, D. J.; Schneider, J. P. Enhanced mechanical rigidity of hydrogels formed from enantiomeric peptide assemblies. J. Am. Chem. Soc., 2011, 133 (38), 14975-14977.

[41] Swanekamp, R. J.; DiMaio, J. T.; Bowerman, C. J.; Nilsson, B. L. Coassembly of enantiomeric amphipathic peptides into amyloidinspired rippled $\beta$-sheet fibrils. J. Am. Chem. Soc., 2012, 134 (12), 5556-5559.

[42] Dzwolak, W.; Ravindra, R.; Nicolini, C.; Jansen, R.; Winter, R. The diastereomeric assembly of polylysine is the low-volume 
pathway for preferential formation of $\beta$-sheet aggregates. J. Am. Chem. Soc., 2004, 126 (12), 3762-3768.

[43] Guilhelmelli, F.; Vilela, N.; Albuquerque, P.; Derengowski, L.; Silva-Pereira, I.; Kyaw, C. Antimicrobial development challenges: The various mechanisms of action of antimicrobial peptides and of bacterial resistance. Front. Microbiol., 2013, 4, 353. doi: 10.3389/fmicb.2013.00353; Wernert, G. T.; Winkler, D. A.; Holan, G.; Nicoletti, G. Synthesis, biological activity, and qsar studies of antimicrobial agents containing biguanide isosteres. Aust. J. Chem., 2004, 57 (1), 77-85

[44] Li, P.; Li, X.; Saravanan, R.; Li, C. M.; Leong, S. S. J. Antimicrobial macromolecules: Synthesis methods and future applications. RSC Adv., 2012, 2 (10), 4031-4044.

[45] Marchesan, S.; Qu, Y.; Waddington, L. J.; Easton, C. D.; Glattauer, V.; Lithgow, T. J.; McLean, K. M.; Forsythe, J. S.; Hartley, P. G. Self-Assembly of ciprofloxacin and a tripeptide into an antimicrobial nanostructured hydrogel. Biomaterials, 2013, 34 (14), 36783687.

[46] Hamley, I. W. The amyloid beta peptide: A chemist's perspective. Role in alzheimer's and fibrillization. Chem. Rev., 2012, 112 (10), 5147-5192.

[47] Krysmann, M. J.; Castelletto, V.; Kelarakis, A.; Hamley, I. W.; Hule, R. A.; Pochan, D. J. Self-Assembly and hydrogelation of an amyloid peptide fragment. Biochem., 2008, 47 (16), 4597-4605.

[48] Li, J.; Kuang, Y.; Gao, Y.; Du, X.; Shi, J.; Xu, B. D-amino acids boost the selectivity and confer supramolecular hydrogels of a nonsteroidal anti-inflammatory drug (nsaid). J. Am. Chem. Soc., 2012, 135 (2), 542-545.

[49] Altunbas, A.; Lee, S. J.; Rajasekaran, S. A.; Schneider, J. P.; Pochan, D. J. Encapsulation of curcumin in self-assembling peptide hydrogels as injectable drug delivery vehicles. Biomaterials, 2011, 32 (25), 5906-5914.

[50] Wang, H.; Wang, Y.; Han, A.; Cai, Y.; Xiao, N.; Wang, L.; Ding, D.; Yang, Z. Cellular membrane enrichment of self-assembling dpeptides for cell surface engineering. ACS Appl. Mater. Interfaces, 2014, 6 (12), 9815-9821.

[51] Fichman, G.; Gazit, E. Self-assembly of short peptides to form hydrogels: Design of building blocks, physical properties and technological applications. Acta Biomater., 2014, 10 (4), 1671-1682.

[52] Yuan, D.; Zhou, R.; Shi, J.; Du, X.; Li, X.; Xu, B. Enzymeinstructed self-assembly of hydrogelators consisting of nucleobases, amino acids, and saccharide. RSC Adv., 2014, 4 (50), 26487-26490.

[53] Hyland, L. L.; Twomey, J. D.; Vogel, S.; Hsieh, A. H.; Yu, Y. B. Enhancing biocompatibility of D-oligopeptide hydrogels by negative charges. Biomacromolecules, 2012, 14 (2), 406-412.

[54] Luo, Z.; Yue, Y.; Zhang, Y.; Yuan, X.; Gong, J.; Wang, L.; He, B.; Liu, Z.; Sun, Y.; Liu, J.; Hu, M.; Zheng, J. Designer D-form selfassembling peptide nanofiber scaffolds for 3-dimensional cell cultures. Biomaterials, 2013, 34 (21), 4902-4913.

[55] Marchesan, S.; Waddington, L.; Easton, C. D.; Kushkaki, F.; McLean, K. M.; Forsythe, J. S.; Hartley, P. G. Tripeptide selfassembled hydrogels: Soft nanomaterials for biological applications. BioNanoSci., 2013, 3 (1), 21-29.

[56] Gahmberg, C. G.; Fagerholm, S. C.; Nurmi, S. M.; Chavakis, T.; Marchesan, S.; Gronholm, M. Regulation of integrin activity and signalling. Biochim. Biophys. Acta, 2009, 1790 (6), 431-444. 